\title{
A Glimpse into the Future of Chronic Wound Healing and Regenerative Medicine Using Self-Assembling Peptides
}

\section{Gayathri Acharya* \\ Department of Product Quality Pharma R\&D, GlaxosmithKline, USA}

*Corresponding author: Gayathri Acharya, Department of Product Quality Pharma R\&D, GlaxosmithKline, 1250 S. Collegeville Road, UP9100, Collegeville, PA 194260989, USA, Tel: 312.731.5644, Email: gaya3acharya@gmail.com

\section{Mini Review \\ Volume 2 Issue 3}

Received Date: August 15, 2018

Published Date: September 05, 2018

\section{Abstract}

Chronic wounds are non-healing wounds, which cease the normal healing process at the inflammatory stage. Diabetic, pressure, venous, and arterial ulcers are the most common chronic wounds. It takes about 2-3 months to identify a nonhealing wound as a chronic wound, which is usually characterized by overactive neutrophils and excess proteases. Current treatment options fail in about $60 \%$ of the cases, leading to severe pain, amputation, and sepsis. Lack of cost effective treatment options and rapidly growing health care costs underline the need for meticulous wound care methods. The definitive treatment for chronic wounds is to provide an acellular dermal matrix that stimulates cellular migration, survival, and proliferation to accelerate wound closure. The purpose of this review is to focus on the recent developments and capabilities of stem cells and self-assembling peptide hydrogels in the process of developing safer and effective wound care.

Keywords: Chronic wound; Cellular Migration; Proliferation; Stem cells; Self-assembling peptide Hydrogels Regenerative tissue

Abbreviations: ECM: Extra Cellular Matrix; ADSCs: Adipose Derived Stem Cells; BMSCs: Bone Marrow mesenchymal Stem Cells; HSPG: Heparin Sulfate Proteoglycan; TNF- $\alpha$ : Tumor Necrosis Factors- $\alpha$; VEGF: Vascular Endothelial Growth Factor; bFGF: Basic Fibroblast Growth Factor; PDGF: Platelet-Derived Growth Factor; IGF: Insulin-like Growth Factor; KGF: Keratinocyte Growth Factor; TGF-c: Transforming Growth Factor; HGF: Hepatocyte Growth Factor; GPx: Glutathione Peroxidase; SODs: Superoxide Dismutase; FGF-2: Fibroblast Growth Factor-2; GCSF: Granulocyte Colony-Stimulating Factor.

\section{Introduction}

Chronic wounds mostly occur in elderly patients who are suffering from major illnesses such as diabetes, obesity, cancer, and heart disease. Treatment of chronic wounds is a major challenge in geriatric medicine. Approximately 6.5 million patients suffer from chronic wounds in the United States alone. Wound care accounts for approximately $\$ 25$ billion in annual costs with an additional $\$ 12$ billion spent on wound scarring $[1,2]$. Compromised immunity, autoimmune diseases, poor diet, and lifestyle often complicate the healing process, leading 


\section{Medicinal \& Analytical Chemistry International Journal}

to amputation, sepsis, and sometimes death. Malnutrition, excessive alcohol consumption, smoking, and poor wound care can further complicate the chronic wound healing process. Dressings, debridement, negative pressure devices, antibiotics, and skin substitutes are the most commonly used initial approaches to wound care. However, these adjuncts are not substitutes for proper wound care. These local adjuncts support the body's repair mechanism and are appropriate for acute wounds. However, chronic wounds require a more integrated approach, targeting both reparative and regenerative processes. To take the integrated approach, a complete understanding of the healing process of complex wounds and wound physiology is required.

The normal wound healing process occurs in four overlapping stages called homeostasis, inflammation, proliferation, and remodeling. Each stage is marked with certain precise cellular events that progress the healing towards the next stage. In hemostasis, the body's defense mechanism immediately constricts the injured blood vessels. A platelet plug is formed at the injured site, followed by fibrin deposition to strengthen the plug. Clot and surrounding wound tissue release certain growth factors and cytokines that mark the onset of the inflammation stage. In the inflammation stage, neutrophils, monocytes, and lymphocytes migrate into the wound to clear microbes, cellular debris, and other contaminants. Monocytes differentiate into macrophages, which release cytokines to further enhance leukocytemediated inflammation. Later, macrophages undergo a phenotypic transition into repair cells that stimulate keratinocytes, fibroblasts, and endothelial cells, promoting the wound into the proliferative stage. During the proliferation stage, fibroblast cells migrate into the wound to form tough granulation tissue. Angiogenic factors released by the fibrin scab stimulate endothelial cell proliferation to form new blood vessels. Enzymes digest fibrin and clot material to accommodate fibroblast migration and angiogenesis simultaneously. Formation of granulation tissue acts as basement tissue that supports epithelial cell proliferation and migration to achieve wound closure. Upon reepithelialization, the woundhealing process enters the final stage, remodeling. This phase is characterized by the continuous remodeling of collagen and other Extracellular Matrix (ECM) materials to reestablish original tissue tensile strength $[3,4]$. Growth factors and cytokines play a major role in mediating all stages of repair process. Depletion of cytokines, growth factors, reduced neovascularization, increased oxidative stress, excessive proteases, cell senesce, and altered immune responses contribute to chronic wounds by affecting the balance between one or more cellular events $[3,5,6]$.

\section{Stem Cells}

\section{Stem Cells and Wound Healing}

Stem cells have self-renewal capacity and differentiate into various cell types based on local stimulation. It was discovered that bone marrow mesenchymal stem cells (BMSCs) accelerate chronic wound healing through differentiation into fibroblasts and synthesis of growth factors $[7,8]$. Although BMSCs have enormous potential in healing processes, obtaining large populations of cells required to elicit the desired therapeutic effect is a major limitation. Adipose derived stem cells (ADSCs) have multilineage cellular plasticity similar to bone marrow stem cells, which makes these cells a good alternative for BMSCs since both these cell lines have similar surface markers and gene profiling. Moreover, ease of isolation, relative abundance, and rapid expansion makes ADSCs favorable for therapeutic use [9]. ADSCs perform multidimensional repair and regeneration of dermal injuries through paracrine and trans-differentiation mechanisms, required for the treatment of chronic wounds. ADSCs have been proven beneficial for wound healing through paracrine and trans-differentiation mechanisms. In ADSCs, integrin $\beta 1$ receptor and heparin sulfate proteoglycan (HSPG) surface protein predominantly regulate cell and fibronectin interaction. Moreover, the heparin-binding domain from fibronectin III domain acts as ligand for various growth factors required for healing application. Therefore, modifications of self-assembling peptides with bioactive synthetic short peptide sequences (motifs) from natural fibronectin proteins are predicted to elicit both cell homing and growth factor sequestering properties.

\section{Paracrine and Antioxidant Effects of Adipose Derived Stem Cells}

Paracrine signaling in ADSC therapy is mediated by the release of cytokines and growth factors, which regulate various cellular pathways required for wound healing. Paracrine signaling protects survival and morphology of fibroblasts under hostile wound conditions. Proteomic analysis of ADSCs secreted growth factors revealed the presence of various growth factors and cytokines required for wound healing [10-12]. Moreover, under the influence of Tumor Necrosis Factors$\alpha$ (TNF- $\alpha$ ), lipopolysaccharides, and severe hypoxia [11], 


\section{Medicinal \& Analytical Chemistry International Journal}

ADSCs the increase release of growth factors (Vascular Endothelial Growth Factor (VEGF) and Basic Fibroblast Growth Factor (bFGF)) [11,13]. Secretion of PlateletDerived Growth Factor (PDGF), Insulin-like Growth Factor (IGF), Keratinocyte Growth Factor (KGF), basic Fibroblast Growth Factor (bFGF), Transforming Growth Factor (TGF-c), Hepatocyte Growth Factor (HGF), VEGF, direct fibroblast, keratinocyte migration, proliferation, and differentiation are crucial for tissue regeneration. Apart from paracrine signaling, ADSCs protect dermal cells from oxidative stress and photo aging [14] caused by chemicals and UV-B $[11,14]$. ADSCs also protect against oxidative stress induced fibroblast cell death by increasing the activity of Glutathione Peroxidase (GPx) and Superoxide Dismutase (SODs), which actively scavenge reactive oxygen species by catalyzing redox reactions [10]. RegranexÒ and Procurenò are the only FDA approved PDGF based medications available for lower extremity diabetic ulcers $[15,16]$. However, exudates from chronic wounds that contain excessive proteases rapidly degrade and clear the growth factors. A novel multi-domain self-assembling peptide hydrogel system with growth factor retaining capability might protect rapid depletion of growth factors by enzymatic degradation while providing controlled release.

\section{Reepithelialization and Angiogenesis}

Reepithelialization is a crucial step for the wound healing process that involves proliferation of keratinocytes at the edges and migration into the middle of the wound [4]. Increased survival, proliferation, and migration of skin keratinocytes promote dermal regeneration in chronic wounds [11]. Angiogenesis is also an important process in the proliferative phase of wound healing, supplying oxygen and other nutrients to newly formed granulation tissue [6]. ADSCs differentiate into epithelial and endothelial cells under the influence of local signals [17]. VEGF and other soluble factors released by local tubular epithelial cells promote epithelial differentiation of ADSCs [17,18]. ADSCs accelerate neovascularization through secretion of angiogenic growth factors like VEGF, HGF and Fibroblast Growth Factor-2 (FGF-2), and direct differentiation into endothelial cells [19]. TGF-b, PDGF-AB, and Granulocyte Colony-Stimulating Factor (GCSF) increase both proliferative and angiogenic potential of ADSCs $[9,20]$. Moreover, under hypoxic conditions, ADSCs enhance stress fiber (actin filaments) formation that provides mechanical contractile force for keratinocyte migration via the ROCK signaling pathway [21]. Based on these observations, a delivery system bracketing both the mechanistic pathways involved in ADSCs' mediated wound healing might fulfill the need for a multidimensional therapeutic approach targeted at various deficits and may accelerate chronic wound healing and tissue regeneration.

\section{Stem Cell Treatment Challenges}

Capturing ADSC paracrine effects requires continuous release of growth factors and cytokines into the wound cells, whereas reepithelialization requires prolonged survival, proliferation, and integration of ADSCs. Hence, in chronic wounds where accelerated repair and large amounts of tissue regeneration is required, a combined approach involving improved ADSC adhesion, survival, proliferation, and slow delivery of ADSC paracrine factors may synergistically enhance chronic wound healing. However, chronic wound healing requires a high concentration of growth factors and stem cells at the wound site. Systemic delivery of stem cells not only delays their transit, but also reduces the number of cells reaching the target site. Systemic toxicity of non-targeted stem cells is a major concern due to the possibility of selfrenewal and differentiation into cancer causing cells. However, topical delivery of stem cells through fibrin spray and collagen sponge promises delivery of a concentrated population of stem cells to the wound site. The problem with this approach is that poor cell survival and retention renders the system ineffective. Moreover, these types of matrix materials have poor mechanical stability and cause immunogenic reactions [22]. In regenerative medicine, interaction between cell binding domain of ECM and cell receptors mediate cell migration, adhesion and cell signal transduction that is crucial for cell functioning [22]. These observations suggest that a topical delivery system with ECM-cell adhesion mimicking properties may improve the challenges that come with stem cell therapy.

\section{Multidomain Self-Assembling Peptide Hydrogels}

Self-assembling peptide hydrogels are a novel class of hydrogels made from peptide sequences that selfassemble into nanostructures. These peptides can be tailored for various purposes including tissue regeneration, proteins, and drug delivery [22-24]. Biodegradable and non-toxic peptide building blocks make them suitable as matrices for controlled protein delivery systems. Furthermore, the high water content of self-assembling peptide hydrogels bio-mimic a 3D 
microenvironment similar to natural ECM that is suitable for cell growth and survival Figure 1.

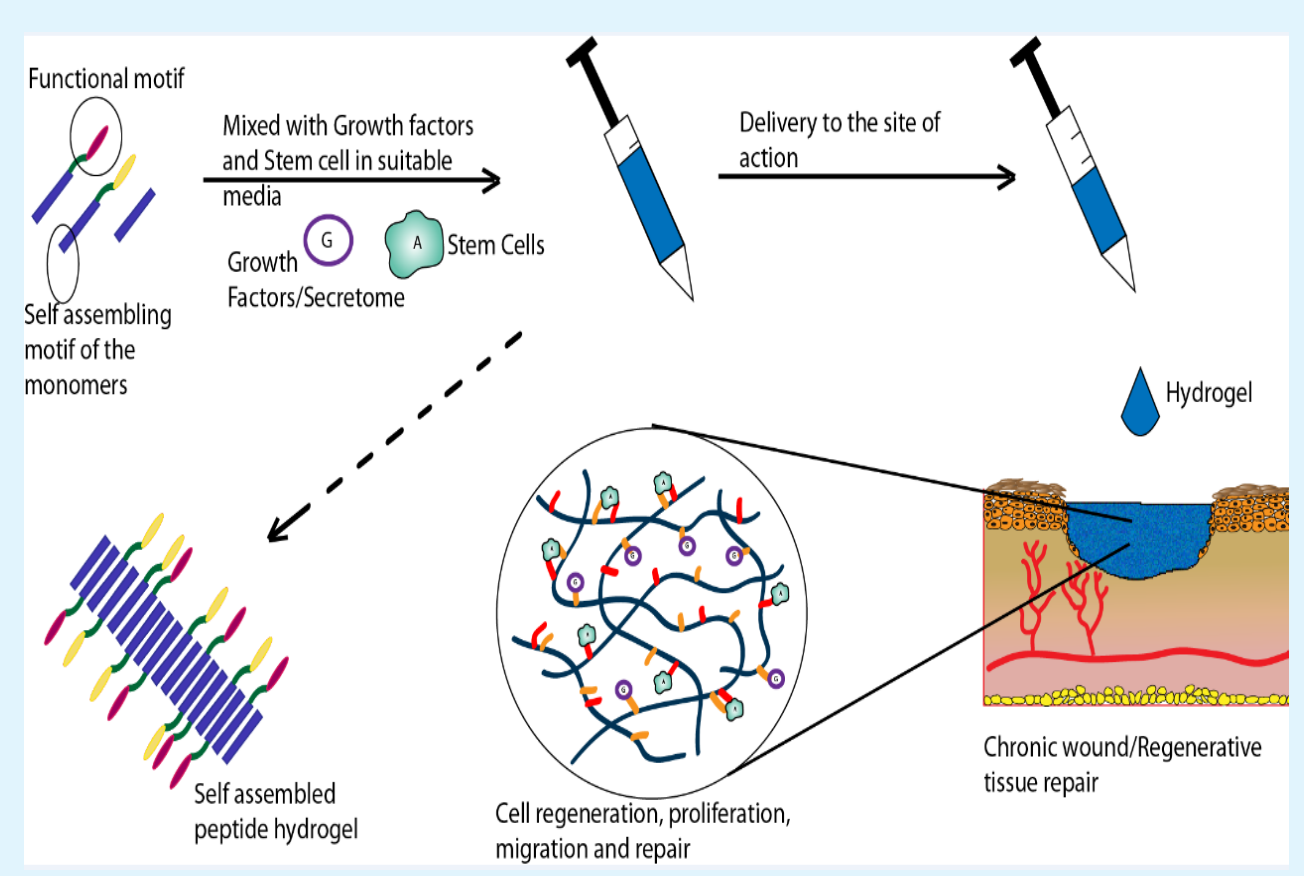

Figure 1: Schematic representing the applicability of self-assembling peptides and stem cells in Chronic wound healing and regenerative medicine.

Self-assembling peptide hydrogels suitable for chronic wound healing should have the following set of desired properties: (i) ability to provide cell-to-hydrogel adhesion; (ii) undergo shear thinning and recover rapidly; (iii) gel formation upon treatment with physiological salts; (iv) resistant to rapid degradation by proteases; (v) offer mechanical strength similar to ECM. The first property pertaining to cell adhesion can be tailored to any self-assembling peptide hydrogels by incorporating cell adhesion motifs (like RGD) into the peptides. Other properties were summarized based on characteristics of chronic wounds and topical delivery systems. A research team from Rice University has developed a few peptide sequences with ABA block motif for various tissueengineering purposes $[23,25]$. Among these, K2 (SL) 6K2 is a mechanically strong peptide sequence that crosses links in the presence of phosphate ions, $\beta$ glycerophosphate, and heparin. K2 (SL) 6K2 peptide hydrogels offer some very unique properties suitable for chronic wound healing. Under high load, they undergo shear thinning followed by rapid recovery within seconds. These observations attest the potential of ADSC-specific peptide (K2 (SL) 6K2) hydrogels in chronic wound healing. Hence, it is hypothesized that K2(SL)6K2 peptide hydrogels, tailored with ADSC receptors binding and growth factor sequestering motifs might enhance stem cells therapy for chronic wound healing.

Hydrogels offer a nourishing 3D microenvironment for stem cell survival, but homing and cell retention requires unique cell adhesion properties. By tailoring specific fragments of ECM proteins to self-assembling peptides, stem cell receptors can be targeted to synthetically mimic the cell-ECM adhesion mechanism. Moreover, several functional peptides (called motifs) attached to selfassembling peptides have been previously combined in varying ratios to elicit specific biological functions on cells [26]. Fibronectin is a multifunctional ECM glycoprotein with cell adhesion and growth factor sequestering domains in its crystal structure $[27,28]$. Fibronectin is deposited along with fibrin in wounds as provisional matrix for reepithelialization and granulation tissue formation [29]. ADSCs predominantly express integrin b1 and Heparin Sulfate Proteoglycan (HSPG) surface receptors that regulate cell adhesion and spreading. Integrin b1 and HSPG ADSC surface receptors 


\section{Medicinal \& Analytical Chemistry International Journal}

synergistically interact with fibronectin III domain for cell adhesion [28,30]. Cell surface HSPG mediates cellfibronectin adhesion through heparin binding domains in the fibronectin protein. Hence, self-assembling peptides designed with integrin and heparin binding functional motifs might provide cues for cell-adhesion and homing. Peptides RGD, PHSRN, PRARI, EILDV, KQAGDV, and REDV are all integrin binding domains present in fibronectin. RGD peptide is the commonly used motif in peptide and polymeric matrices for cell adhesion. RGD and PHSRN motif act both synergistically and independently to mediate integrin a5b1 receptor binding $[31,32]$. PRARI is a new integrin-binding motif whose potential is yet to be fully explored [28,33]. Heparin binding domains on fibronectin have been attributed to PRRARV, WQPPRARI, SPPRRARVI, and LIGRKK sequences. WQPPRARI is the most effective sequence with PRARI peptide retaining majority of its activity [34].

Along with providing a 3D microenvironment, the capability to capture ADSC secreted growth factors is a major challenge due to poor yield and the large number of stem cells required for the extraction process. Additionally, proteolytic degradation and rapid clearance of growth factors are the major limitations for growth factor treatment in chronic wounds. Application of ECM proteins to interact with growth factors sequesters and protects the growth factors, producing controlled release [35]. Also it has been noted that heparin-binding domains in Fibronectin III12-14 shows have high binding affinity with growth factors from diverse families [36]. Application of fibronectin III12-14 sequences to selfassembling peptides might accentuate growth factor bioactivity. WQPPRARI and PRARI peptide sequence discussed earlier are also known heparin-binding motifs from fibronectin III12-14 domains. Therefore, selfassembling peptide hydrogels designed with heparin binding motifs (C2-C5) may play dual role of cell adhesion and growth factor sequestering. Controlled release of growth factors from multidomain hydrogels can be modified by controlling ratio of heparin binding sequence in the hydrogel formation [37-40].

From the discussion above, we can anticipate that designer hydrogels will not only provide a moisturebalanced matrix for fibroblast survival and migration, but also provide growth factor sequestering and stem cell homing microenvironment. We hypothesize that dual acting multi domain self-assembling peptide hydrogels designed to deliver both paracrine and trans differentiation based ADSC therapy might synergistically accelerate chronic wound healing without the need for multiple dosages.

\section{Potential Impact}

a) The proposed multidomain hydrogel system can potentially have an immense clinical, economical, and scientific impact especially in geriatric patient care.

b) Hydrogels can serve as a platform for stem cell based therapies and regenerative medicine where accessing the target site and multiple dosing are the major limitations.

c) Topical application of stem cells with self-assembling peptide hydrogels will overcome systemic toxicity associated with stem cell therapy.

d) Accelerated wound closure attenuates scar formation reducing the need for expensive cosmetic surgeries.

e) The synergistic effect of growth factors and stem cells combined therapy may be applied to stem cell based therapies where high population of stem cells are required to elicit the required function.

f) Proposed delivery systems may serve as a platform for controlled growth factor, proteins, and peptide delivery.

\section{References}

1. KC Steven Percival (2010) Microbiology of Wounds. (1 ${ }^{\text {st }}$ Edn).

2. Sen CK, Gordillo GM, Roy S, Kirsner R, Lambert L, et al. (2009) Human skin wounds: a major and snowballing threat to public health and the economy. Wound repair and regeneration 17(6): 763-771.

3. Guo S, Dipietro LA (2010) Factors affecting wound healing. Journal of dental research 89(3): 219-229.

4. Hunt TK, Hopf H, Hussain Z (2000) Physiology of wound healing. Advances in skin \& wound care 13(2): 6-11.

5. Jun JI, Lau LF (2010) Cellular senescence controls fibrosis in wound healing. Aging 2(9): 627-631.

6. Nie C, Yang D, Morris SF (2009) Local delivery of adipose-derived stem cells via acellular dermal matrix as a scaffold: a new promising strategy to accelerate wound healing. Medical hypotheses 72(6): 679-682. 


\section{Medicinal \& Analytical Chemistry International Journal}

7. Fathke C, Wilson L, Hutter J, Kapoor V, Smith A, et al. (2004) Contribution of bone marrow-derived cells to skin: collagen deposition and wound repair. Stem cells 22(5): 812-822.

8. Han SK, Yoon TH, Lee DG, Lee MA, Kim WK (2005) Potential of human bone marrow stromal cells to accelerate wound healing in vitro. Annals of plastic surgery 55(4): 414-419.

9. De Rosa, F De Francesco, V Tirino, GA Ferraro, V Desiderio, et al. (2009) A new method for cryopreserving adipose-derived stem cells: an attractive and suitable large-scale and long-term cell banking technology. Tissue engineering Part C Methods 15(4): 659-667.

10. Kim WS, Park BS, Kim HK, Park JS, Kim KJ, et al. (2008) Evidence supporting antioxidant action of adipose-derived stem cells: protection of human dermal fibroblasts from oxidative stress. Journal of dermatological science 49(2): 133-142.

11. Kim WS, Park BS, Sung JH (2009) The wound-healing and antioxidant effects of adipose-derived stem cells. Expert opinion on biological therapy 9(7): 879-887.

12. Lynch SE, Colvin RB, Antoniades HN (1989) Growth factors in wound healing. Single and synergistic effects on partial thickness porcine skin wounds. The Journal of clinical investigation 84(2): 640-646.

13. Lee EY, Xia Y, Kim WS, Kim MH, Kim TH, et al. (2009) Hypoxia-enhanced wound-healing function of adipose-derived stem cells: increase in stem cell proliferation and up-regulation of VEGF and bFGF. Wound repair and regeneration 17(4): 540-547.

14. Kim WS, Park BS, Sung JH, Yang JM, Park SB, et al. (2007) Wound healing effect of adipose-derived stem cells: a critical role of secretory factors on human dermal fibroblasts. Journal of dermatological science 48(1): 15-24.

15. Harding KG, Morris HL, Patel GK (2002) Science, medicine and the future: healing chronic wounds. BMJ 324(7330): 160-163.

16. Senet $P$ (2004) [Becaplermin gel (Regranex gel)]. Annales de dermatologie et de venereologie 131(4): 351-358.
17. Baer PC (2011) Adipose-derived stem cells and their potential to differentiate into the epithelial lineage. Stem cells and development 20(10): 1805-1816.

18. Cao Y, Sun Z, Liao L, Meng Y, Han Q, et al. (2005) Human adipose tissue-derived stem cells differentiate into endothelial cells in vitro and improve postnatal neovascularization in vivo. Biochemical and biophysical research communications 332(2): 370379.

19. Lu F, Mizuno H, Uysal CA, Cai X, Ogawa R, et al. (2008) Improved viability of random pattern skin flaps through the use of adipose-derived stem cells. Plastic and reconstructive surgery 121(1): 50-58.

20. Lin $\mathrm{YF}$, Jing $\mathrm{W}, \mathrm{Wu} \mathrm{L}, \mathrm{Li} \mathrm{XY}, \mathrm{Wu} \mathrm{Y}$, et al. (2008) Identification of osteo-adipo progenitor cells in fat tissue. Cell proliferation 41(5): 803-812.

21. Moon KM, Park YH, Lee JS, Chae YB, Kim MM, et al. (2012) The effect of secretory factors of adiposederived stem cells on human keratinocytes. International journal of molecular sciences 13(1): 1239-1257.

22. Zhu J, Marchant RE (2011) Design properties of hydrogel tissue-engineering scaffolds. Expert review of medical devices 8(5): 607-626.

23. Bakota EL, Wang Y, Danesh FR, Hartgerink JD (2011) Injectable multidomain peptide nanofiber hydrogel as a delivery agent for stem cell secretome. Biomacromolecules 12(5): 1651-1657.

24. Horii, Wang X, Gelain F, Zhang S (2007) Biological designer self-assembling peptide nanofiber scaffolds significantly enhance osteoblast proliferation, differentiation and 3-D migration. PloS one 2(2): e 190.

25. Galler KM, Aulisa L, Regan KR, D'Souza RN, Hartgerink JD (2010) Self-assembling multidomain peptide hydrogels: designed susceptibility to enzymatic cleavage allows enhanced cell migration and spreading. Journal of the American Chemical Society 132(9): 3217-3223.

26. Gelain F, Bottai D, Vescovi A, Zhang S (2006) Designer self-assembling peptide nanofiber scaffolds for adult mouse neural stem cell 3-dimensional cultures. PloS one 1(1): e119. 


\section{Medicinal \& Analytical Chemistry International Journal}

27. Martino MM, Tortelli F, Mochizuki M, Traub S, BenDavid D, et al. (2011) Engineering the growth factor microenvironment with fibronectin domains to promote wound and bone tissue healing. Science translational medicine 3(100): 100ra89.

28. Sharma A, Askari JA, Humphries MJ, Jones EY, Stuart DI (1999) Crystal structure of a heparin- and integrinbinding segment of human fibronectin. The EMBO journal 18(6): 1468-1479.

29. Lin F, Ren XD, Pan Z, Macri L, Zong WX, et al. (2011) Fibronectin growth factor-binding domains are required for fibroblast survival. The Journal of investigative dermatology 131(1): 84-98.

30. Park S, Han M, Rhie JW, Kim SH, Jung Y, et al. (2009) The correlation between human adipose-derived stem cells differentiation and cell adhesion mechanism. Biomaterials 30(36): 6835-6843.

31. Craig JA, Rexeisen EL, Mardilovich A, Shroff K, Kokkoli E (2008) Effect of linker and spacer on the design of a fibronectin-mimetic peptide evaluated via cell studies and AFM adhesion forces. Langmuir: the ACS journal of surfaces and colloids 24(18): 10282-10292.

32. Feng Y, Mrksich M (2004) The synergy peptide PHSRN and the adhesion peptide RGD mediate cell adhesion through a common mechanism. Biochemistry 43(50): 15811-15821.

33. Chen H, Yuan L, Song W, Wu Z, Li D (2008) Biocompatible polymer materials: Role of proteinsurface interactions. Progress in Polymer Science 33(11): 1059-1087.

34. Woods A, McCarthy JB, Furcht LT, Couchman JR (1993) A synthetic peptide from the COOH-terminal heparin-binding domain of fibronectin promotes focal adhesion formation. Molecular biology of the cell 4(6): 605-613.

35. Wang B, Wu Y, Shao Z, Yang S, Che B, et al. (2012) Functionalized self-assembling peptide nanofiber hydrogel as a scaffold for rabbit nucleus pulposus cells. Journal of biomedical materials research Part A 100: 646-653.

36. Martino MM, Hubbell JA (2010) The 12th-14th type III repeats of fibronectin function as a highly promiscuous growth factor-binding domain. FASEB journal 24(12): 4711-4721.

37. Kopesky PW, Vanderploeg EJ, Kisiday JD, Frisbie DD, Sandy JD (2011) Controlled delivery of transforming growth factor beta1 by self-assembling peptide hydrogels induces chondrogenesis of bone marrow stromal cells and modulates Smad2/3 signaling. Tissue engineering Part A 17(1-2): 83-92.

38. Segers VF, Lee RT (2007) Local delivery of proteins and the use of self-assembling peptides. Drug discovery today $12(13-14)$ : 561-568.

39. Zhao Y, Tan T, Yokoi H, Tanaka M, Kinoshita T (2008) Controlled release and interaction of protein using self-assembling peptide RATEA16 nanofiber hydrogels. Journal of Polymer Science Part A: Polymer Chemistry 46(14): 4927-4933.

40. Koutsopoulos S, Unsworth LD, Nagai Y, Zhang S (2009) Controlled release of functional proteins through designer self-assembling peptide nanofiber hydrogel scaffold. Proceedings of the National Academy of Sciences of the United States of America 106(12): 4623-4628.



Gayathri Acharya. A Glimpse into the Future of Chronic Wound Healing and Regenerative Medicine Using Self-Assembling Peptides. Med \& Analy Chem Int J 2018, 